\title{
Author Index to Volume 37 - 2010
}

\begin{tabular}{|c|c|c|c|c|c|c|c|}
\hline Abu-Arafeh, I. & 769 (NOV) & Bilbao, J.M. & 416 (MAY) & Cooper, P.E. & 449 (JULY) & Fang, C. & 240 (MAR) \\
\hline Ahmed, S. & 252 (MAR) & Bilo, L. & 376 (MAY) & & 553 (SEPT) & Farb, R. & 412 (MAY) \\
\hline Ahmed, S.N. & 814 (NOV) & Binns, M.A. & 601 (SEPT) & Cortez, M.A. & $656(\mathrm{SEPT})$ & Fazl, M. & 416 (MAY) \\
\hline Akbar, N. & 608 (SEPT) & Bitar, R. & 498 (JULY) & Cossette, $\mathrm{P}$. & $819(\mathrm{NOV})$ & Fehlings, M.G. & 49 (JAN) \\
\hline Algethamy, $\mathrm{H}$. & $897(\mathrm{NOV})$ & Blacquiere, D. & 866 (NOV) & Couillard, P.L. & $568(\mathrm{SEPT})$ & & 271 (MAR) \\
\hline Al-Hashmi, A. & 684 (SEPT) & Blevins, G. & S3 (SEPT) & Crockford, D. & 473 (JULY) & Feinstein, A. & 608 (SEPT) \\
\hline Ali, A. & 515 (JULY) & Bock, J.E. & $138(\mathrm{JAN})$ & Crumley, P. & 808 (NOV) & Feldman, H.H. & 359 (MAY) \\
\hline Ali, $S$. & 293 (MAR) & Bojanowski, M.W. & 219 (MAR) & Cubero-González, A. & $692(\mathrm{SEPT})$ & Findlay, J.M. & $310($ MAY) \\
\hline Aliya, N. & 177 (MAR) & & 283 (MAR) & Curran, $\mathbf{M}$. & $135(\mathrm{JAN})$ & & 320 (MAY) \\
\hline Al-Jehani, $\mathrm{H}$. & $671(\mathrm{SEPT})$ & & 402 (MAY) & da Costa, L. & 286 (MAR) & & 541 (JULY) \\
\hline Alkhajawah, M. & 716 (NOV) & & 881 (NOV) & D'Alfonso, S. & 457 (JULY) & & 843 (NOV) \\
\hline Al Khalidi, H. & 416 (MAY) & Bolton, C.F. & 431 (JULY) & Darsaut, T.E. & $861(\mathrm{NOV})$ & Fineman, I. & $125(\mathrm{JAN})$ \\
\hline \multirow[t]{2}{*}{ AlKhawajah, M. } & 305 (MAY) & Bouchard, T. & 595 (SEPT) & Da Silva, V.F. & 273 (MAR) & Flood, T. & 524 (JULY) \\
\hline & 307 (MAY) & Boulanger, J-M. & 791 (NOV) & & 904 (NOV) & Foroghipoor, $\mathrm{M}$. & 371 (MAY) \\
\hline Al-Khotani, A. & 868 (NOV) & Boulos, M.I. & 167 (MAR) & Davey, P. & $36(\mathrm{JAN})$ & Fourney, D.R. & 535 (JULY) \\
\hline \multirow[t]{2}{*}{ Almekhlafi, M.A. } & 512 (JULY) & & 878 (NOV) & Davis, $\mathrm{K}$. & 135 (JAN) & & 719 (NOV) \\
\hline & 568 (SEPT) & Brahim, E.M. & 515 (JULY) & Deacon, $\mathrm{C}$. & $826(\mathrm{NOV})$ & Fournier, M.R. & $121(\mathrm{JAN})$ \\
\hline Al-Radi, O.O. & $412(\mathrm{MAY})$ & Breen, $\mathrm{K}$. & 904 (NOV) & Dehdashti, A.R. & 532 (JULY) & Fox, AJ. & 498 (JULY) \\
\hline Alvarez, G.G. & 904 (NOV) & Brodeur, C. & 336 (MAY) & Deis, N. & 843 (NOV) & Fox, S.H. & $86(\mathrm{JAN})$ \\
\hline Andrade, D.M. & $141(\mathrm{JAN})$ & Brotchie, J.M. & 86 (JAN) & Del Bigio, M.R. & 206 (MAR) & Frangou, E. & 703 (SEPT) \\
\hline Ang, C. & 625 (SEPT) & Brown, L. & 615 (SEPT) & & 725 (NOV) & Fraser, F.C. & 304 (MAY) \\
\hline Angel, $\mathrm{M}$. & $141(\mathrm{JAN})$ & Burneo, J.G. & 868 (NOV) & DeMarchi, R. & $416(\mathrm{MAY})$ & Fraser, J.A. & 88 (JULY) \\
\hline Antel, J. & S42 (SEPT) & Bussière, M. & $866(\mathrm{NOV})$ & Deslauriers, A. & S24 (SEPT) & Freedman, M. & 601 (SEPT) \\
\hline Antony, J.M. & S24 (SEPT) & Butcher, K.S. & $4(\mathrm{JAN})$ & Devkota, U.P. & 76 (JAN) & Frisoni, G.B. & 67 (JAN) \\
\hline Auer, R.N. & 213 (MAR) & & 17 (JAN) & Diab, S. & $110(\mathrm{JAN})$ & Fulvia de Leva, M. & 376 (MAY) \\
\hline \multirow[t]{5}{*}{ Aviv, R.I. } & $36(\mathrm{JAN})$ & & 320 (MAY) & Díaz-Nicolás, S. & 692 (SEPT) & Furtado, S. & 512 (JULY) \\
\hline & 276 (MAR) & & $808(\mathrm{NOV})$ & Di Fabio, R. & 406 (MAY) & Galluzzi, S. & 67 (JAN) \\
\hline & 498 (JULY) & Butler, R. & 359 (MAY) & Ding, $P$. & 643 (SEPT) & Gao, $\mathrm{P}$. & 697 (SEPT) \\
\hline & $681(\mathrm{SEPT})$ & Cabrera-Naranjo, F. & $692(\mathrm{SEPT})$ & Dion, Y. & $110(\mathrm{JAN})$ & Garant, M-P. & 791 (NOV) \\
\hline & 878 (NOV) & Camicioli, R. & $595(\mathrm{SEPT})$ & Dominguez-González, & & Gariépy, J-L. & 492 (JULY) \\
\hline Azarpazhooh, M.R. & $37 \mathrm{I}(\mathrm{MAY})$ & Campbell, C. & 195 (MAR) & & 269 (MAR) & Gaspar, C. & $110(\mathrm{JAN})$ \\
\hline Banerjee, T.K. & 637 (SEPT) & Cao, S-Z. & 365 (MAY) & Dowlatshahi, D. & $866(\mathrm{NOV})$ & Gauthier, S. & 2 (JAN) \\
\hline \multirow[t]{2}{*}{ Barnett, H.J.M. } & 160 (MAR) & Cao, $\mathrm{Y}$. & 697 (SEPT) & Drake, B. & 408 (MAY) & Gawel, M. & 588 (SEPT) \\
\hline & $562(\mathrm{SEPT})$ & Carrier, N. & $791(\mathrm{NOV})$ & Duan, $\mathbf{H}$. & 240 (MAR) & Gee, M. & 595 (SEPT) \\
\hline Baronia, B. & 482 (JULY) & Casali, C. & 406 (MAY) & Dubé, M-P. & $110(\mathrm{JAN})$ & Geroldi, C. & 67 (JAN) \\
\hline Bartlett, E.S. & 498 (JULY) & Casaubon, L.K. & $412(\mathrm{MAY})$ & Duggal, N. & 650 (SEPT) & Ghani, U. & 797 (NOV) \\
\hline Beattie, B.L. & $359(\mathrm{MAY})$ & Castrioto, A. & 478 (JULY) & & 837 (NOV) & Gharavi, M.M. & 371 (MAY) \\
\hline Beaudoin, O.X. & 881 (NOV) & Chaabouni, A. & 279 (MAR) & Dunham, C. & 213 (MAR) & Giammarco, R. & 588 (SEPT) \\
\hline Beaulieu, C. & $595(\mathrm{SEPT})$ & Chakraborty, D.P. & 637 (SEPT) & Dupré, N. & $302(\mathrm{MAY})$ & Gil, R. & 468 (JULY) \\
\hline \multirow[t]{3}{*}{ Becker, W.J. } & 449 (JULY) & Chambers, B.R. & 371 (MAY) & Duquette, $\mathrm{P}$. & $551(\mathrm{SEPT})$ & Gill, H.S. & 631 (SEPT) \\
\hline & $580(\mathrm{SEPT})$ & Charland, D.A. & 54 (JAN) & Durand-Martel, P. & 336 (MAY) & Gillis, C. & 855 (NOV) \\
\hline & $588(\mathrm{SEPT})$ & Chen, G-P. & 803 (NOV) & Dwosh, E. & 359 (MAY) & Girard, M-E. & 819 (NOV) \\
\hline Bekhor, S. & 888 (NOV) & Chen, G-Z. & 803 (NOV) & Earl, A. & 849 (NOV) & Girgis, $\mathrm{F}$. & 855 (NOV) \\
\hline Bélair, M. & 881 (NOV) & Chen, S-D. & 229 (MAR) & Ebers, G.C. & 912 (NOV) & Giuliani, F. & S3 (SEPT) \\
\hline Bell, $R$. & $512(J U L Y)$ & Chen, Z. & 504 (JULY) & Ebrahim, S. & $49(\mathrm{JAN})$ & Gladstone, J. & 553 (SEPT) \\
\hline Benstead, T.J. & 419 (MAY) & Chhibber, S. & 517 (JULY) & & $855(\mathrm{NOV})$ & & 588 (SEPT) \\
\hline Berger M.J. & $54(\mathrm{JAN})$ & Chinchilla, M. & 831 (NOV) & Elbers, J. & 909 (NOV) & Glikstein, R.S. & 684 (SEPT) \\
\hline Bernat, J.L. & 758 (NOV) & Chittock, D.R. & 43 (JAN) & Émond, F. & 395 (MAY) & & 904 (NOV) \\
\hline \multirow[t]{4}{*}{ Bernstein, $M$. } & 49 (JAN) & Chouinard, S. & $110(\mathrm{JAN})$ & Edmondson, E.A. & 389 (MAY) & Goez, H.R. & 96 (JAN) \\
\hline & 293 (MAR) & Chow, T.W. & 601 (SEPT) & Ellestad, K.K. & S24 (SEPT) & Goldstein, W. & 681 (SEPT) \\
\hline & 422 (MAY) & Christie, S.D. & $131(\mathrm{JAN})$ & El Matri, L. & 279 (MAR) & Gomez-Ramirez, J. & 86 (JAN) \\
\hline & 706 (SEPT) & Christie, S.N. & 449 (JULY) & Emery, D. & $4(\mathrm{JAN})$ & González-Hernández, A & \\
\hline Berthelet, F. & 402 (MAY) & & $588(\mathrm{SEPT})$ & Emery, D. & $17(\mathrm{JAN})$ & & 692 (SEPT) \\
\hline Bhalerao, S. & 901 (NOV) & Chudy, D. & 118 (JAN) & Erendzhinova, E. & 528 (JULY) & Gourdeau, A. & 890 (NOV) \\
\hline \multirow[t]{2}{*}{ Bharatha, A. } & 498 (JULY) & Cieuta-Walti, C. & 826 (NOV) & Fabre-Pi, O. & 692 (SEPT) & Goyal, M. & 849 (NOV) \\
\hline & 681 (SEPT) & Claessens, C. & 890 (NOV) & Fallah, A. & $49(\mathrm{JAN})$ & Grewal, R.P. & 521 (JULY) \\
\hline Bharmal, A. & 473 (JULY) & Colantonio, A. & $783(\mathrm{NOV})$ & & 271 (MAR) & Griebel, R.W. & 223 (MAR) \\
\hline Bhat, $\mathrm{R}$. & S24 (SEPT) & Concha, L. & 595 (SEPT) & & 855 (NOV) & Griesdale, D.E.G. & $43(\mathrm{JAN})$ \\
\hline Biernaskie, J. & S34 (SEPT) & Cooke, L.J. & $580(\mathrm{SEPT})$ & Fan, J.C. & 245 (MAR) & Guimond, C. & 359 (MAY) \\
\hline
\end{tabular}




\section{Author Index to Volume 37 - 2010}

\begin{tabular}{|c|c|c|c|c|c|c|c|}
\hline Guidetti, V. & 769 (NOV) & Kalokhe, U. & 289 (MAR) & Lu, X-D. & $803(\mathrm{NOV})$ & Morrow, S.A. & 488 (JULY) \\
\hline \multirow[t]{3}{*}{ Guiot, M-C. } & 289 (MAR) & Kalra, S. & 687 (SEPT) & Ludwin, S.K. & 159 (MAR) & Mozeg, D. & 276 (MAR) \\
\hline & 625 (SEPT) & Kamal, A.K. & 252 (MAR) & Lum, C. & $135(\mathrm{JAN})$ & Mubrin, Z. & $118(\mathrm{JAN})$ \\
\hline & 671 (SEPT) & Katramados, A.M. & 700 (SEPT) & & 866 (NOV) & Mukherjee, S.C. & 637 (SEPT) \\
\hline Habek, M. & $118(\mathrm{JAN})$ & Kavan, $\mathrm{P}$. & 625 (SEPT) & Lysenko, L. & 521 (JULY) & Munoz, D. & 289 (MAR) \\
\hline Hader, W.J. & $28(\mathrm{JAN})$ & Kazim, S.F. & 258 (MAR) & $\mathrm{Ma}, \mathrm{H}$. & 365 (MAY) & Munshi, A. & 177 (MAR) \\
\hline Haji, F. & 855 (NOV) & Keane, J.R. & 662 (SEPT) & $\mathrm{Ma}, \mathrm{W}$. & 521 (JULY) & Murray, B.J. & 167 (MAR) \\
\hline Hajnšek, S. & 118 (JAN) & & 870 (NOV) & Macaulay, R.J. & $131(\mathrm{JAN})$ & Murtaza, M. & 252 (MAR) \\
\hline Hamani, C. & $141(\mathrm{JAN})$ & Keith-Rokosh, J. & 620 (SEPT) & Macdonald, R.L. & 289 (MAR) & Murthy, S.B. & 138 (JAN) \\
\hline Hameed, B. & 258 (MAR) & Khan, K. & 808 (NOV) & Machado, C. & 831 (NOV) & Mysak, T.M. & 245 (MAR) \\
\hline Hammond, R.R. & 620 (SEPT) & Khan, Muh. & 700 (SEPT) & Mackie, G. & 449 (JULY) & Nabalamba, A. & 186 (MAR) \\
\hline $\mathrm{Hao}, \mathrm{S}-\mathrm{C}$. & 365 (MAY) & Khan, Mus. & 258 (MAR) & & 588 (SEPT) & Nataraj, A. & 1 (JAN) \\
\hline Harder, S. & 703 (SEPT) & Khoury, M.A. & 54 (JAN) & Mah, J.K. & 195 (MAR) & Nguyen, D.K. & 219 (MAR) \\
\hline Hatef, M.R. & 371 (MAY) & Khu, K.J. & 293 (MAR) & Main, C.D. & 457 (JULY) & & 819 (NOV) \\
\hline Hawkins, C. & 873 (NOV) & & 863 (NOV) & Malak, R. & 219 (MAR) & Nguyen, O.F. & 684 (SEPT) \\
\hline Hayley, S. & 555 (SEPT) & Kiberd, M.B. & $131(\mathrm{JAN})$ & Mamdani, M. & 383 (MAY) & Nicolle, D. & 488 (JULY) \\
\hline Heran, M.K.S. & $128(\mathrm{JAN})$ & Kilty, S. & 402 (MAY) & Mann, C. & 814 (NOV) & Nicolle, D.A. & 837 (NOV) \\
\hline Hershey, A.D. & 769 (NOV) & Koizumi, T. & 113 (JAN) & Mann, S.A. & 535 (JULY) & & 897 (NOV) \\
\hline Hicks, T.P. & 457 (JULY) & Kolski, H. & 279 (MAR) & Marchak, E. & $320(\mathrm{MAY})$ & Niu, Z-F. & 365 (MAY) \\
\hline \multirow[t]{2}{*}{ Hill, M.D. } & 397 (MAY) & Kraft, S.P. & 631 (SEPT) & Marcoux, J. & 671 (SEPT) & Noël de Tilly, L. & 574 (SEPT) \\
\hline & $568(\mathrm{SEPT})$ & Kremenchutzky, M. & 488 (JULY) & Marin, S.A. & 273 (MAR) & Noorbakhsh, F. & S24 (SEPT) \\
\hline Hinnell, C. & 512 (JULY) & Kurth, T. & 43 (JAN) & Massicotte, E.M. & $49(\mathrm{JAN})$ & Novick, $R$. & 897 (NOV) \\
\hline Hoffmann, G.F. & 350 (MAY) & Kus, L.H. & 650 (SEPT) & Matouk, C.C. & 532 (JULY) & Nzau, $M$ & 482 (JULY) \\
\hline Houeto, J.L. & 468 (JULY) & Labib, M.A. & 837 (NOV) & McAndrews, M-P. & 141 (JAN) & O'Connor, P.W. & $98(\mathrm{JAN})$ \\
\hline Howard, P. & 286 (MAR) & Laforce Jr., R. & $302(\mathrm{MAY})$ & McDonald, $\mathrm{P}$. & 301 (MAY) & & 383 (MAY) \\
\hline Howe, $\mathrm{K}$. & 855 (NOV) & Lahijani, E. & 389 (MAY) & & 400 (MAY) & & 608 (SEPT) \\
\hline Hsiung, G-Y.R. & 359 (MAY) & Lam, K. & $36(\mathrm{JAN})$ & McEwen, J. & 43 (JAN) & Oger, J. & 305 (MAY) \\
\hline $\mathrm{Hu}, \mathbf{M}$. & $643(\mathrm{SEPT})$ & Lang, A.E. & 478 (JULY) & McKenzie, M. & 264 (MAR) & & 307 (MAY) \\
\hline $\mathrm{Hu}, \mathrm{Z}$. & 643 (SEPT) & Langevin, $\mathrm{S}$. & 492 (JULY) & McLachlan, R.S. & 557 (SEPT) & & $716(\mathrm{NOV})$ \\
\hline Hulliger, $\mathrm{S}$. & 478 (JULY) & Lavigne, F. & 402 (MAY) & McLaughlin, N. & 283 (MAR) & Ohinmaa, A. & 814 (NOV) \\
\hline Hunter, G. & 235 (MAR) & Leblanc, N. & $791(\mathrm{NOV})$ & & 402 (MAY) & O'Kelly, C.J. & 320 (MAY) \\
\hline \multirow[t]{2}{*}{ Huot, $P$. } & 86 (JAN) & Lee, D. & 837 (NOV) & McMillan, H.J. & 195 (MAR) & & 549 (SEPT) \\
\hline & 395 (MAY) & Lee, L. & 276 (MAR) & Megyesi, J. & $620($ SEPT) & & 681 (SEPT) \\
\hline Ibrahim, G.M. & 855 (NOV) & & 878 (NOV) & Mehta, V. & 677 (SEPT) & Olson, R. & 264 (MAR) \\
\hline \multirow[t]{2}{*}{ Ikeda, K.M. } & 779 (NOV) & Lemire, E.G. & 528 (JULY) & & 723 (NOV) & Orouji, E. & 371 (MAY) \\
\hline & $893(\mathrm{NOV})$ & Lesiuk, $\mathrm{H}$. & 906 (NOV) & Menascu, S. & 656 (SEPT) & Otani, R. & 703 (SEPT) \\
\hline Islam, M. & 258 (MAR) & Lespérance, $\mathrm{P}$. & $110(\mathrm{JAN})$ & Menon, B.K. & 397 (MAY) & Pablo-Fernández, E.D. & 269 (MAR) \\
\hline \multirow[t]{3}{*}{ Jacob, F.D. } & $96(\mathrm{JAN})$ & Létourneau, K. & $826(\mathrm{NOV})$ & Meo, R. & 376 (MAY) & Paci, M. & 888 (NOV) \\
\hline & 694 (SEPT) & Leuzy, A. & $2(\mathrm{JAN})$ & Merims, D. & $601(\mathrm{SEPT})$ & Pandya, A. & $568(\mathrm{SEPT})$ \\
\hline & $885(\mathrm{NOV})$ & Lévêque, $\mathrm{M}$. & 881 (NOV) & Metz, L.M. & 615 (SEPT) & Paquet, N. & 336 (MAY) \\
\hline Jacobsen, K.X. & 457 (JULY) & Lewis, S.J. & $49(\mathrm{JAN})$ & Meyer, M. & $620(\mathrm{SEPT})$ & Paquette, F. & 861 (NOV) \\
\hline Jaglal, S. & 783 (NOV) & Lewko, J. & 783 (NOV) & Michaud, J. & 524 (JULY) & Parent, A. & 313 (MAY) \\
\hline Jan, M.M. & 439 (JULY) & $\mathrm{Li}, \mathrm{J}$ & 873 (NOV) & Midha, R. & 863 (NOV) & Parent, M. & $313(\mathrm{MAY})$ \\
\hline Jasinska-Myga, B. & $61(\mathrm{JAN})$ & $\mathrm{Li}, \mathrm{M}$ & 240 (MAR) & Miller, F.D. & S34 (SEPT) & Parkinson, M. & 264 (MAR) \\
\hline Javidan, M. & 721 (NOV) & Lian, J. & 574 (SEPT) & Mitchell, J.R. & 615 (SEPT) & Parks, N.E. & 419 (MAY) \\
\hline \multirow[t]{2}{*}{ Jawaid, A. } & $138(\mathrm{JAN})$ & Limaiem, F. & 279 (MAR) & & 849 (NOV) & Parrent, A. & 868 (NOV) \\
\hline & 389 (MAY) & Limaiem, R. & 279 (MAR) & Modi, J. & 849 (NOV) & Patten, S.B. & 186 (MAR) \\
\hline \multirow[t]{2}{*}{ Jeerakathil, T.J. } & 245 (MAR) & Links, K.A. & 601 (SEPT) & Mohamed, I. & 656 (SEPT) & Payne, K.B. & 457 (JULY) \\
\hline & 808 (NOV) & Lipsman, N. & 412 (MAY) & Mohammad, A. & 797 (NOV) & Pearson, G.J. & 245 (MAR) \\
\hline Jenkins, M.E. & $868(\mathrm{NOV})$ & Listrat, A. & 468 (JULY) & Mohammed, B. & 515 (JULY) & Peddareddygari, L.R. & 521 (JULY) \\
\hline Jobin, C. & 551 (SEPT) & Liu, Z-T. & 365 (MAY) & Mokhber, N. & 371 (MAY) & Perez-Nellar, J. & 831 (NOV) \\
\hline \multirow[t]{2}{*}{ Johnson, E.S. } & 206 (MAR) & Lobaugh, N.J. & 608 (SEPT) & Moldzio, R. & 81 (JAN) & Perry, J. & 36 (JAN) \\
\hline & 687 (SEPT) & Lohani, S. & 76 (JAN) & Montanera, W. & 574 (SEPT) & Pfeffer, G. & $128(\mathrm{JAN})$ \\
\hline Johnston, W.S.W. & 687 (SEPT) & López-Veloso, A.C. & 692 (SEPT) & Moore, G.R.W. & S5 (SEPT) & Phillips, J.T. & $54(\mathrm{JAN})$ \\
\hline Johnston, T. & $86(\mathrm{JAN})$ & Lord, S. & 343 (MAY) & Moradzadeh, L. & $608(\mathrm{SEPT})$ & Pierelli, F. & 406 (MAY) \\
\hline Joseph, J.T. & 512 (JULY) & Loungarath, V. & $890(\mathrm{NOV})$ & Moretti, P. & 389 (MAY) & Pirouzmand, F. & 276 (MAR) \\
\hline Juurlink, B.H.J. & 223 (MAR) & Lowry, N.J. & 528 (JULY) & Moro, E. & 478 (JULY) & Pluchon, C. & 468 (JULY) \\
\hline Kalia, L.V. & 276 (MAR) & $\mathrm{Lu}, \mathrm{C}$. & 473 (JULY) & Morrish, W.F. & 568 (SEPT) & Poon, Y-Y. & 478 (JULY) \\
\hline Kalia, S.K. & 276 (MAR) & Lu, G-Q. & 229 (MAR) & Morrison, M. & 36 (JAN) & Porteri, C. & $67(\mathrm{JAN})$ \\
\hline
\end{tabular}




\section{Author Index to Volume 37 - 2010}

\begin{tabular}{|c|c|c|c|c|c|c|c|}
\hline Poublanc, J. & $141(\mathrm{JAN})$ & Salamone, A.R. & 389 (MAY) & Stevens, C. & 574 (SEPT) & Wang, Fe. & 240 (MAR) \\
\hline Poulter, M.O. & 457 (JULY) & Saliba, I. & $881(\mathrm{NOV})$ & St. George-Hyslop, P. & 359 (MAY) & Wang, G. & 229 (MAR) \\
\hline \multirow[t]{2}{*}{ Power, C. } & S3 (SEPT) & Salman, M.S. & 779 (NOV) & St-Onge, J. & $110(\mathrm{JAN})$ & Wang, S. & 697 (SEPT) \\
\hline & S24 (SEPT) & & 893 (NOV) & Strong, M.J. & 54 (JAN) & Wang, S-J. & 769 (NOV) \\
\hline Prasad, A.N. & 350 (MAY) & Salmon, F. & 468 (JULY) & Stys, P.K. & S16 (SEPT) & Wang, Z-Q. & 229 (MAR) \\
\hline Puangthong, U. & 359 (MAY) & Sanghera, $P$. & $36(\mathrm{JAN})$ & Suchowersky, $O$. & 213 (MAR) & Weaver, D.F. & 539 (JULY) \\
\hline Purgina, $\mathrm{B}$. & 524 (JULY) & Sanyal, J. & 637 (SEPT) & & 473 (JULY) & Wei, C.W. & 574 (SEPT) \\
\hline Putzke, J.D. & 61 (JAN) & Saqqur, $\mathrm{M}$ & 808 (NOV) & Sun, $M$. & 873 (NOV) & Weill, A. & 861 (NOV) \\
\hline Quickfall, J. & 473 (JULY) & Sarkar, B. & 637 (SEPT) & Sun, $X$. & 240 (MAR) & Wein, T.H. & 888 (NOV) \\
\hline Quon, M.J. & $54(\mathrm{JAN})$ & Sarnat, H.B. & $105(\mathrm{JAN})$ & Suzuki, Y. & $113(\mathrm{JAN})$ & Weir, B. & 745 (NOV) \\
\hline Qureshi, S.U. & $138(\mathrm{JAN})$ & Savard, M. & 492 (JULY) & Swaine, B. & 783 (NOV) & Wells, S. & 343 (MAY) \\
\hline Racine, E. & 758 (NOV) & & 890 (NOV) & Syeda, U-E-R. & 252 (MAR) & Wennberg, R. & 141 (JAN) \\
\hline Radad, K. & 81 (JAN) & Saverino, $\mathrm{C}$. & 783 (NOV) & Symons, S.P. & $36(\mathrm{JAN})$ & Wheatley, M.B. & 885 (NOV) \\
\hline Radic, J. & 855 (NOV) & Schellenberg, K.L. & 687 (SEPT) & & 276 (MAR) & White, J.H. & 498 (JULY) \\
\hline Rahay, S.R. & 439 (JULY) & Scherle, C. & 831 (NOV) & & 498 (JULY) & Whittaker, T. & 808 (NOV) \\
\hline Rajbhandari, H. & $76(\mathrm{JAN})$ & Schipper, H.M. & 684 (SEPT) & & 681 (SEPT) & Wider, C. & 61 (JAN) \\
\hline Rajput, $\mathrm{M}$. & 235 (MAR) & Schmidt, B.J. & $121(\mathrm{JAN})$ & Tabei, Y. & $113(\mathrm{JAN})$ & Wiebe, S. & $814(\mathrm{NOV})$ \\
\hline Ramanakumar, A.V. & $625(\mathrm{SEPT})$ & Schültke, E. & 223 (MAR) & Taj, F. & 252 (MAR) & Wilkinson, J.S. & 535 (JULY) \\
\hline \multirow[t]{2}{*}{ Ramaswamy, V. } & $96($ JAN $)$ & Schulz, P.E. & 138 (JAN) & Tam, S. & 837 (NOV) & Willinsky, R.A. & 286 (MAR) \\
\hline & 694 (SEPT) & & 389 (MAY) & Tan, Y-Y. & 229 (MAR) & & 532 (JULY) \\
\hline Rampersaud, Y.R. & $49(\mathrm{JAN})$ & Schulz, V.M. & 54 (JAN) & Tao, H-M. & 803 (NOV) & & 681 (SEPT) \\
\hline Rao, V.R. & 637 (SEPT) & Schwindt, $\mathbf{B}$. & 797 (NOV) & Tator, C.H. & 343 (MAY) & Wilson, J.R. & $873(\mathrm{NOV})$ \\
\hline Rapoport, M.J. & 276 (MAR) & Scott, C.J.M. & 608 (SEPT) & & 714 (NOV) & Wiltshire, K.M. & 213 (MAR) \\
\hline Rausch, W.D. & $81(\mathrm{JAN})$ & Sebag, M. & 861 (NOV) & Teitelbaum, J. & 429 (JULY) & & 595 (SEPT) \\
\hline Ray, B.C. & 637 (SEPT) & Sedighi, S. & 371 (MAY) & & 713 (NOV) & Winner, $\mathrm{P}$. & 769 (NOV) \\
\hline Reddy, $\mathrm{H}$. & 620 (SEPT) & Selchen, D. & 574 (SEPT) & Tellez-Zenteno, J.F. & 309 (MAY) & Wöber-Bingöl, C. & 769 (NOV) \\
\hline Redekop, G.J. & 408 (MAY) & Seshia, S.S. & 769 (NOV) & Tellier, G. & $110(\mathrm{JAN})$ & Wollmann, R. & 289 (MAR) \\
\hline Reid, S. & 213 (MAR) & & 914 (NOV) & ter Brugge, $\mathrm{K}$. & 286 (MAR) & Wong, C.L. & $901(\mathrm{NOV})$ \\
\hline \multirow[t]{2}{*}{ Resch, L. } & 677 (SEPT) & Shafi, G. & 177 (MAR) & & 532 (JULY) & Wong, J.H. & 568 (SEPT) \\
\hline & 687 (SEPT) & Shafqat, S. & 258 (MAR) & Thompson, A.L. & 498 (JULY) & Wrogemann, J. & 893 (NOV) \\
\hline Rewcastle, N.B. & 725 (NOV) & Shahideh, M. & 855 (NOV) & Torres, C. & 671 (SEPT) & $\mathrm{Wu}, \mathrm{C}$. & 04 (JULY) \\
\hline Reyes, G. & 86 (JAN) & Shamji, M.F. & 482 (JULY) & Toth, C. & 517 (JULY) & Wszolek, Z.K. & 61 (JAN) \\
\hline Rezaieyazdi, Z. & 371 (MAY) & & 524 (JULY) & Tremblay, D. & 336 (MAY) & Xia, H-C. & 365 (MAY) \\
\hline Ribeiro, M. & 286 (MAR) & & $906(\mathrm{NOV})$ & Trépanier, $\mathrm{P}$. & 890 (NOV) & Xiao, L. & 643 (SEPT) \\
\hline Ricard, G. & 791 (NOV) & Shao, B. & 803 (NOV) & Trottier, F. & 492 (JULY) & $\mathrm{Xu}, \mathrm{D}$. & 240 (MAR) \\
\hline Richer, F. & $110(\mathrm{JAN})$ & Sharma, K. & 849 (NOV) & Tsao, M.N. & 36 (JAN) & Xue, L. & 643 (SEPT) \\
\hline Riopelle, R. & 758 (NOV) & Sharma, K.B. & 904 (NOV) & Tshechmer, S.M. & 656 (SEPT) & Yagi, K. & $113(\mathrm{JAN})$ \\
\hline Ritsma, B.R. & 54 (JAN) & Sharma, P. & 512 (JULY) & Tsukada, M. & 113 (JAN) & Yamada, R. & $113(\mathrm{JAN})$ \\
\hline Rivière, J-B. & $110(\mathrm{JAN})$ & Shemie, S.D. & $758(\mathrm{NOV})$ & Tu, A. & 677 (SEPT) & Yeo, T. & 677 (SEPT) \\
\hline Roberge, D. & 625 (SEPT) & Shinoura, N. & $113(\mathrm{JAN})$ & Turgeon, A.F. & 492 (JULY) & Young, G.B. & 157 (MAR) \\
\hline \multirow[t]{2}{*}{ Robinson, C.A. } & 528 (JULY) & Shirani, $\mathbf{P}$. & 389 (MAY) & Tynan, J. & 703 (SEPT) & & 429 (MAY) \\
\hline & 535 (JULY) & Shobha, N. & 568 (SEPT) & Uitti, R.J. & 61 (JAN) & & 713 (NOV) \\
\hline Robinson, D.B. & 121 (JAN) & Shroff, M. & 656 (SEPT) & Vachhrajani, S. & 873 (NOV) & & 897 (NOV) \\
\hline Rodrigue, $\mathrm{C}$. & 758 (NOV) & Shuaib, A. & 797 (NOV) & Vadalà, R. & 406 (MAY) & Zabad, R. & 615 (SEPT) \\
\hline Rogaeva, E. & 359 (MAY) & & 808 (NOV) & Vandermarcq, $P$ & 468 (JULY) & Zagorski, B. & 783 (NOV) \\
\hline Ross, I.B. & $125(\mathrm{JAN})$ & Silver, F. & 717 (NOV) & Van Uum, S. & 837 (NOV) & Zahid, R. & 252 (MAR) \\
\hline Rotenberg, B. & 837 (NOV) & Simpson, $M$. & 849 (NOV) & Vassilyadi, M. & 82 (JULY) & Zairi, I. & 279 (MAR) \\
\hline Rotenberg, B.W. & $650(\mathrm{SEPT})$ & Sinclair, D.B. & 885 (NOV) & Velayati, A. & 371 (MAY) & Žarković, K. & 118 (JAN) \\
\hline Rotstein, D.L. & 383 (MAY) & Singh, J. & 397 (MAY) & Ventureyra, C.G. & 482 (JULY) & Zeiler, F.A. & 400 (MAY) \\
\hline Rouleau, G.A. & $110(\mathrm{JAN})$ & Singh, N. & 681 (SEPT) & & 524 (JULY) & Zhang, B. & 240 (MAR) \\
\hline Rutka, J.T. & 873 (NOV) & Skinner, C.R. & 273 (MAR) & Vernich, L. & 783 (NOV) & Zhang, Y. & 615 (SEPT) \\
\hline Ryu, W.H.A. & $837(\mathrm{NOV})$ & Smyth, P. & S3 (SEPT) & Visanji, N. & $86(\mathrm{JAN})$ & Zhao, J. & 697 (SEPT) \\
\hline Sacher, A. & 289 (MAR) & Song, X. & 504 (JULY) & Voll, C. & 235 (MAR) & Zhao, S. & 504 (JULY) \\
\hline Sadler, M. & 439 (JULY) & Staines, W.A. & 457 (JULY) & & 703 (SEPT) & Zhou, H-Y. & 229 (MAR) \\
\hline Sadovnick, A.D. & 359 (MAY) & St. Amand, H. & 904 (NOV) & Wallace, M.C. & 532 (JULY) & Zinman, L. & 3 (JAN) \\
\hline Sahgal, A. & 36 (JAN) & Steiner, J.P. & 457 (JULY) & & 681 (SEPT) & & \\
\hline Saint-Hilaire, J-M. & 819 (NOV) & Steinke, D. & 677 (SEPT) & & 855 (NOV) & & \\
\hline Saito, A. & 897 (NOV) & Steinman, $\mathrm{L}$. & S49 (SEPT) & Wang, Fa. & 365 (MAY) & & \\
\hline
\end{tabular}

